\title{
IL-23 produced by CNS-resident cells controls $T$ cell encephalitogenicity during the effector phase of experimental autoimmune encephalomyelitis
}

\author{
Burkhard Becher, ${ }^{1,2}$ Brigit G. Durell, ${ }^{2}$ and Randolph J. Noelle ${ }^{2}$ \\ ${ }^{1}$ Department of Neurology, Neuroimmunology Unit, University of Zurich, Zurich, Switzerland \\ ${ }^{2}$ Department of Microbiology and Immunology, Dartmouth College, Lebanon, New Hampshire, USA
}

\begin{abstract}
CNS-resident cells, in particular microglia and macrophages, are a source of inflammatory cytokines during inflammation within the CNS. Expression of IL-23, a recently discovered cytokine, has been shown to be critical for the development of experimental autoimmune encephalomyelitis (EAE) in mice. Expression of the p40 subunit of IL-12 and IL-23 by microglia has been shown in situ and in vitro, but direct evidence for a functional significance of $\mathrm{p} 40$ expression by CNS cells during an immune response in vivo is still lacking. Here we report that $\mathrm{p} 40$ plays a critical role in maintaining encephalitogenicity during the disease course. By using irradiation bone marrow chimeras, we have generated mice in which $\mathrm{p} 40$ is deleted from the CNS parenchyma but not the systemic immune compartment. Our studies show that $\mathrm{p} 40$ expressed by CNS-endogenous cells is critical for the development of myelin oligodendrocyte glycoprotein-induced EAE. In spite of the reduced clinical disease, the absence of $\mathrm{p} 40$ from the CNS has little impact on the degree of inflammation. Expression profiles of the CNS lesions show an increase in Th2 cytokines when compared with mice that develop EAE in the presence of CNS IL-12 and/or IL-23. Taken together, our data demonstrate that p40 expression by CNS-resident cells forms the basis for the Th1 bias of the CNS.
\end{abstract}

J. Clin. Invest. 112:1186-1191 (2003). doi:10.1172/JCI200319079.

\begin{abstract}
Introduction
The last year has witnessed a major change in focus regarding the role of IL-12 in inflammation. Once considered a major mediator in the development of experimental autoimmune encephalomyelitis (EAE), it has now been reported by a number of laboratories that IL-12 is not essential in the development of EAE or of a number of other inflammatory disorders (1-3). Confusion as to the role of IL-12 in inflammation arose due to the use of the common IL-12 p40 subunit by a previously unknown cytokine, IL-23 (4). IL-12 is a $70-\mathrm{kDa}$ heterodimer consisting of a p35 and a p40 subunit (5). Recently we and others have shown that the IL-12 p40 subunit is indeed required for the induction of EAE in mice, but that the p35 subunit and thus IL-12 was not necessary for disease induction via active immunization with myelin oligodendrocyte glycoprotein (MOG) peptide in CFA (1). The explanation for this surprising observation was that another $\mathrm{p} 40$-containing cytokine was critical for EAE. In this context, IL-23, a newly iden-
\end{abstract}

Received for publication May 30, 2003, and accepted in revised form August 12, 2003.

Address correspondence to: Burkhard Becher, Department of Neurology, Neuroimmunology Unit, University Hospital of Zurich, Nord1D, Room 231, Postfach 38, Frauenklinikstrasse 10, 8091 Zurich, Switzerland. Phone: 41-0-1-255-8842;

Fax: 41-0-1-255-9765; E-mail: burkhard.becher@usz.ch.

Conflict of interest: The authors have declared that no conflict of interest exists.

Nonstandard abbreviations used: experimental autoimmune encephalomyelitis (EAE); myelin oligodendrocyte glycoprotein (MOG); phycoerythrin (Pe). tified $\mathrm{p} 40 / \mathrm{p} 19$ heterodimeric cytokine, has been shown to be the critical mediator in the pathogenesis of EAE. Indeed, $p 19^{-/-}$mice have recently been demonstrated to be completely resistant to the induction of $\mathrm{MOG}_{35-55}$ peptide-induced EAE, confirming that IL-23 rather than IL-12 is responsible for the generation of encephalitogenic cells (3).

The dramatic changes in our understanding of the role of IL-12 and IL-23 in EAE have generated many new questions. During the effector phase of MS and in $\mathrm{EAE}$, cells residing in the CNS have been shown to produce $\mathrm{p} 40$. Windhagen et al. found the p 40 subunit of IL-12 and IL-23 produced by macrophages/microglia in MS plaques (6). During EAE, p40 is expressed within the CNS, and its levels appear to correlate with disease severity (7). In vitro, both human and murine microglia have been shown to produce $\mathrm{p} 40$ as well as bioactive IL-12 in response to engagement of CD40 or toll-like receptors $(8-10)$. However, at this time there is no compelling evidence for a functional role of microglia-derived p40 in EAE. Cua and coworkers have shown that resident microglia and infiltrating macrophages express mRNA for $\mathrm{p} 19$, and have further shown that virus-mediated expression recombinant of IL-23 within the CNS can support EAE in $p 19^{-/-}$mice (3). These observations pose the important question of whether production of IL-23 by microglial cells or by CNS-infiltrating macrophages is critical to the development of this inflammatory disease.

In this report, we present data demonstrating that p40 produced within the CNS by resident microglial cells is critical for conserving the encephalitogenicity 


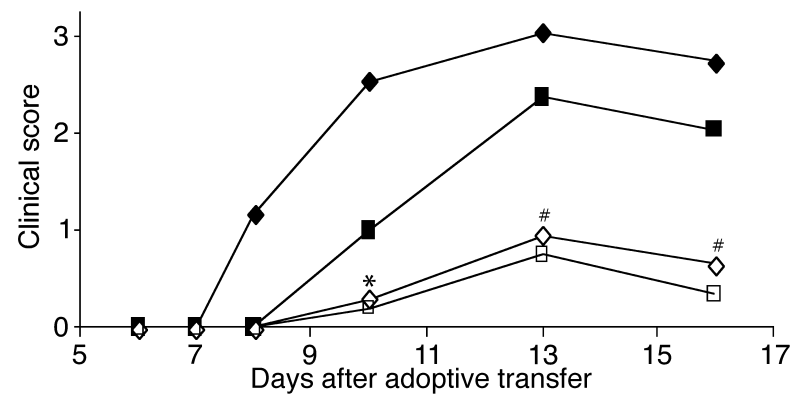

Figure 1

Adoptive transfer of MOG-reactive lymphocytes into WT and $p 40^{-/-}$ recipients. MOG-reactive lymphocytes were generated in WT mice and harvested as described. Cells $\left(2.5 \times 10^{7}\right.$, represented by diamonds, or $1.25 \times 10^{7}$, represented by squares) were injected into WT (filled symbols) or $p 40^{-1-}$ (open symbols) recipients. Clinical disease was monitored, and the difference between WT and $p 40^{-/-}$mice 3 days after disease onset was significant $\left({ }^{*} P<0.05,{ }^{\#} P<0.01\right)$ as assessed using a nonpaired Student's $t$ test. Data shown are from one representative experiment of at least three individual experiments with at least five mice per group.

in a population of MOG peptide-reactive lymphocytes. By using irradiation BM chimeras, we have generated mice in which p40 is deleted from the CNS parenchyma but not the systemic immune compartment. Our studies show that p40 expressed by CNSendogenous cells is critical for the development of MOG-induced EAE.

\section{Methods}

Mice. Female C57BL/6 and CD45 congenic C56BL/ 6-Ly5.2 mice were obtained from the National Cancer Institute (Frederick, Maryland, USA). Homozygous IL-12 $p 35^{-/-}$and IL-12 $p 40^{-/-}$C57BL/ 6 mice were originally purchased from The Jackson Laboratory (Bar Harbor, Maine, USA) and bred in-house under pathogen-free conditions.

Irradiation BM chimeric mice. BM-donor mice were euthanized using $\mathrm{CO}_{2}$ and $\mathrm{BM}$ cells were isolated by flushing femur and tibia bones with HBSS. BM was filtered through a $100-\mu \mathrm{m}$ cell strainer and cells were washed with BSS. CD45 congenic recipient mice were lethally irradiated (12 Gy in a split dose) and intravenously injected with $2 \times 10^{7} \mathrm{BM}$ cells. Engraftment took place over 6-8 weeks of recovery. Mice were bled retro-orbitally to ensure more than $95 \%$ engraftment of blood leukocytes. We have previously reported that this protocol allows us to generate mice in which the
APC compartment of the CNS and of the immune system can be completely separated (11).

Peptides and Ab's. MOG $35-55$ peptide (MEVGWYRSPFSRVVHLYRNGK) was obtained from Research Genetics (Huntsville, Alabama, USA). For cytofluorometric analysis, the antibodies against the following were used: CD45-phycoerythrin (CD45-Pe), CD11b-FITC (Mac1), CD4-biotin, GR-1-biotin, CD8-biotin, and NK1.1-biotin. Biotin labels were visualized using streptavidin-allophycocyanin (Pharmingen, La Jolla, California, USA). All Ab's were obtained from Pharmingen.

Induction of EAE. For active immunization, 5- to 8week-old female C57BL/6 or $p 40^{-/-}$mice (13- to 16 week-old BM chimeric mice) were immunized subcutaneously with $200 \mu \mathrm{g}$ of $\mathrm{MOG}_{35-55}$ peptide emulsified in CFA supplemented with $2 \mathrm{mg} / \mathrm{ml}$ of Mycobacterium tuberculosis (Difco Laboratories, Detroit, Michigan, USA). The mice received intraperitoneal injections with $250 \mathrm{ng}$ pertussis toxin (Sigma-Aldrich, St. Louis, Missouri, USA) at the time of immunization and 48 hours later. After 7 days, the mice received an identical booster immunization with MOG peptide in CFA without pertussis toxin. Clinical disease usually commences between day 12 and day 18 after immunization.

For adoptive transfer, donor mice were immunized subcutaneously with $200 \mu \mathrm{g} \mathrm{MOG}_{35-55}$ in CFA supplemented with $500 \mu \mathrm{g}$ Mycobacterium tuberculosis. Immediately after immunization and 2 days later, the mice received $250 \mathrm{ng}$ of pertussis toxin. Eleven days after immunization, the mice were sacrificed, spleens were removed and homogenized, and red blood cells were lysed. The cells were cultured for 4 days in RPMI 1640 supplemented with $10 \%$ FCS (both obtained from BioWhittaker Inc., Walkersville, Maryland, USA), $10 \mu \mathrm{g} / \mathrm{ml}$ of MOG peptide, and $2.5 \mathrm{ng} / \mathrm{ml}$ of recombinant IL-12 (PeproTech Inc., Rocky Hill, New Jersey, USA). The cells were harvested, and dead cells were removed by Ficoll (Sigma-Aldrich) centrifugation. Cells were then washed and injected into recipient mice $\left(5 \times 10^{6}\right.$ to $2.5 \times 10^{7}$ cells/mouse $)$. Animals received $250 \mathrm{ng} /$ mouse pertussis toxin on day 0 and day 2 after transfer. Clinical disease usually commences 6-10 days after cell transfer.

Clinical evaluation. The mice were scored four times per week as follows: 0 , no detectable signs of EAE; 0.5, distal limp tail; 1, complete limp tail; 1.5 , limp tail and hind limb weakness; 2 , unilateral partial hind-limb paralysis; 2.5 , bilateral partial hind-limb paralysis; 3 , complete bilateral hind-limb paralysis; 3.5 , complete

Table 1

EAE induced by adoptive transfer of encephalitogenic lymphocytes

\begin{tabular}{lcc} 
Group & \multicolumn{2}{c}{ Incidence } \\
$\mathrm{WT}+2.5 \times 10^{7} \mathrm{MOG}-$ reactive cells & $6 / 6$ & $100 \%$ \\
$\mathrm{WT}+1.25 \times 10^{7} \mathrm{MOG}-$ reactive cells & $5 / 6$ & $83.33 \%$ \\
$p 40^{-/-}+2.5 \times 10^{7} \mathrm{MOG}-$ reactive cells & $2 / 5$ & $40.00 \%$ \\
$p 40^{-/-}+1.25 \times 10^{7} \mathrm{MOG}-$-reactive cells & $2 / 5$ & $40.00 \%$
\end{tabular}

Mean day of disease onset ${ }^{\mathrm{A}}$

8.3

11.2

11.5

11.5
Mean maximal clinical score ${ }^{A}$

3.0

2.9

2.4

1.9

ADiseased animals only. 

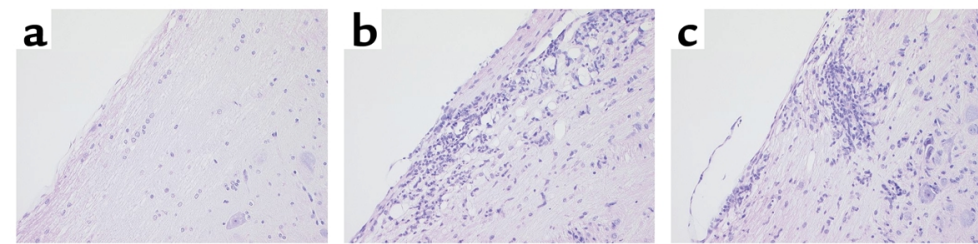

Figure 2

Infiltration of leukocytes into the CNS of WT and $p 40^{-1-}$ mice. Animals were sacrificed 5 days after disease onset, and spinal cord inflammation was assessed by H\&E staining (magnification, $\times 100)$. (a) Naive WT control. Adoptively transferred WT (b) or $p 40^{-/-}$(c) recipients.

hind-limb paralysis and unilateral forelimb paralysis; 4 , total paralysis of fore and hind limbs (score greater than 4 to be euthanized); 5 , death.

Histology. Mice were euthanized with $\mathrm{CO}_{2}$. They were then perfused with PBS and the spinal column of each mouse was removed and fixed in $10 \%$ buffered formalin. The spinal cord was dissected and paraffin embedded prior to staining with $\mathrm{H} \& \mathrm{E}$ to assess infiltration. Flow cytometry. Mice were euthanized with $\mathrm{CO}_{2}$ and spinal cords were removed by flushing the spinal column with sterile BSS. The brain was dissected to isolate the brain stem. Both tissues were homogenized and strained through a $100-\mu \mathrm{m}$ nylon filter (Fisher Scientific Co., Pittsburgh, Pennsylvania, USA). After centrifugation, the cell suspension was resuspended in $37 \%$ isotonic Percoll and underlaid with $70 \%$ isotonic Percoll. The gradient was centrifuged at $600 \mathrm{~g}$ for 25 minutes at room temperature. The interphase cells were collected and extensively washed prior to staining. For flow cytometry, the cells were stained with primary Ab's for 30 minutes at $4^{\circ} \mathrm{C}$, washed, and incubated with streptavidin-allophycocyanin (Pharmingen) for $15 \mathrm{~min}$ utes. The cells were washed and analyzed using a FACSCalibur system and CellQuest software (Becton, Dickinson and Co., San Jose, California, USA). Data analysis was performed using WinMDI 2.8 software (Scripps Research Institute, La Jolla, California, USA).

Recall responses. Mice were primed by flank injections of MOG pep-

\section{Figure 3}

a
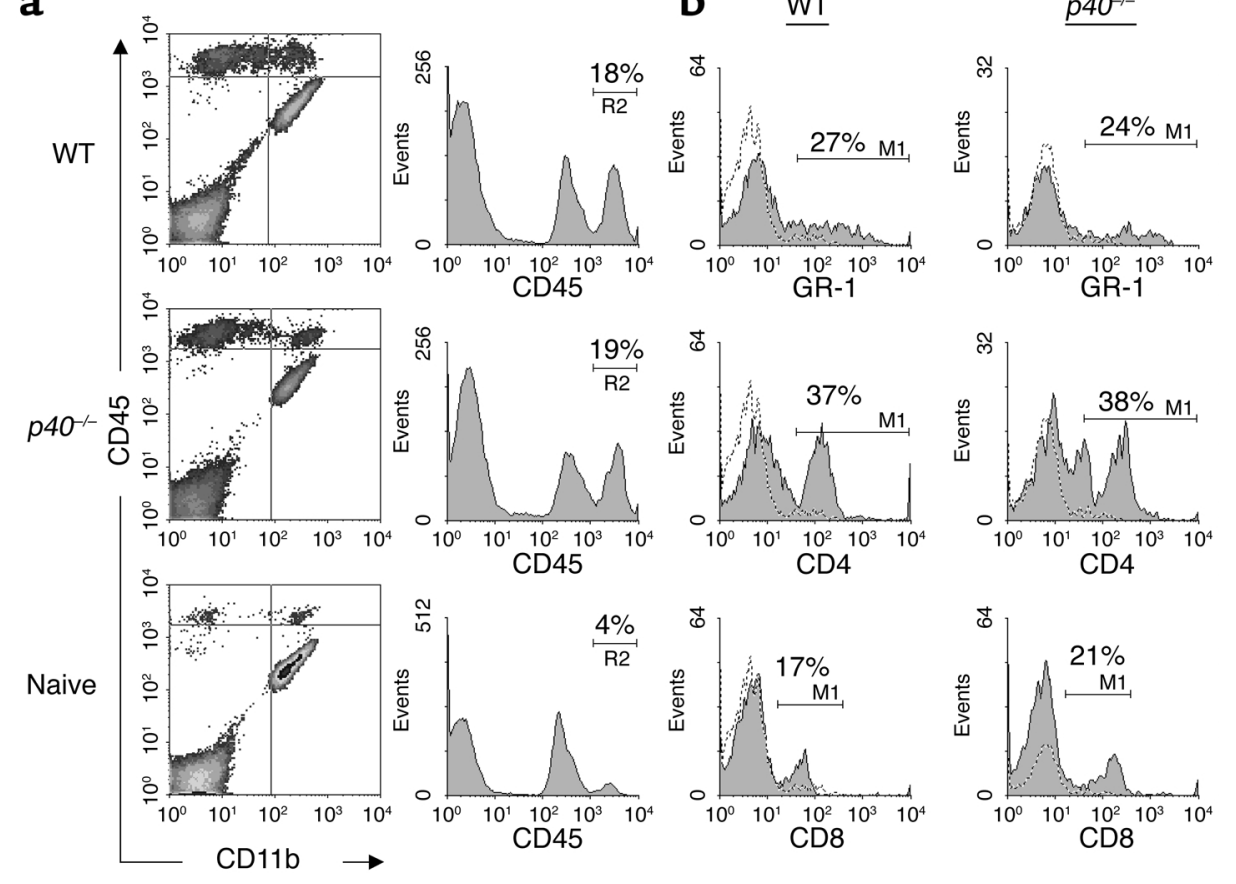

b
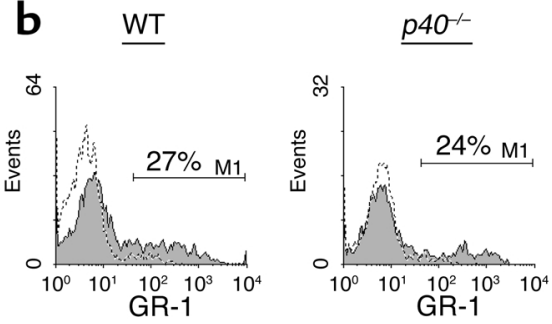

ration was assessed using a Filtermate harvester and a TopCount NXT microplate scintillation and luminescence counter (both from Packard Bioscience Co., Meriden, Connecticut, USA). For cytokine analysis, sister cultures were harvested 48 hours later, and culture supernatants were analyzed by ELISA for IFN- $\gamma$ and IL-2 (Pharmingen).

Real-time PCR. RNA was extracted from spinal cords of mice with EAE as described (11). Briefly, the spinal column was flushed with ice-cold HBSS and the cords were homogenized in Trizol reagent (Invitrogen Corp., Carlsbad, California, USA). RNA was extracted following the manufacturer's instructions. The samples were tide in CFA. After 5 days, the axillary and homogenized. Lymph node cells $\left(5 \times 10^{5}\right)$ were placed in triplicate in a 96-well plate and pulsed with different amounts of myelin proteolipid protein (PLP $139-151$; Research Genetics) as a control. After 48 hours, cells were pulsed with $\left[{ }^{3} \mathrm{H}\right]$ thymidine (NEN Life Science Products, Boston, Massachusetts, USA) and incubated for an additional 15 hours before cells were harvested. Thymidine incorpo-

Inflammatory makeup of infiltrating cells. Animals were sacrificed at day 24 after injection with encephalitogenic lymphocytes, and spinal cord inflammation was assessed by flow cytometry. Infiltrates were stained with antibodies against CD45, CD11b, CD4, CD8, and GR-1 as shown above. (a) Density plots of CNS cells double-stained with CD45 and CD11b. Histograms show staining with CD45-Pe. The marker M1/R2 represents CNS-infiltrating cells. (b) Histograms of CNS-infiltrating cells (R2) isolated from WT and $p 40^{-1-}$ mice injected with encephalitogenic lymphocytes. The filled histograms show staining with anti-GR-1, anti-CD4, and anti-CD8 mAb's. The dotted histograms represent the appropriate isotype controls. Percentage of positive cells is shown above the marker line (M1). 
Table 2

Phenotype of BM chimeras

\begin{tabular}{lcc}
\hline BM donor & Recipient mouse & p40 expression \\
WT & WT & Whole body \\
WT & $p 40^{-/-}$ & Immune compartment \\
$p 40^{-/-}$ & $\mathrm{WT}$ & CNS parenchyma \\
$p 40^{-/-}$ & $p 40^{-/-}$ & Null \\
\hline
\end{tabular}

treated with DNase using DNA AWAY (Molecular Bioproducts Inc., San Diego, California, USA), and $5 \mu \mathrm{g}$ of RNA was transcribed into cDNA using oligo-dT primers and the SuperScript II RT kit (Invitrogen Corp.). Twelve nanograms cDNA per well was transferred into iCycler 96-well plates (Bio-Rad Laboratories Inc., Hercules, California, USA), and SuperMix-UDG master mix containing Taq polymerase (Invitrogen Corp.) was added according to the manufacturer's instructions. The following primer-probe pairs were used: GATA-3: sense, AGAACCGGCCCCTTATCAA; antisense, AGTTCGCGCAGGATGTCC; probe, CCAAGCGAAGGCTGTCGGCAG. IFN- $\gamma$ : sense, GCATTCATGAGTATTGCCAAG; antisense, GGTGGACCACTCGGATGA; probe, ACCCACAGGTCCAGCGCCA. IL-4: sense, ACAGGAGAAGGGACGCCAT; antisense, GAAGCCCTACAGACGAGCTCA; probe, TCCTCACAGCAACGAAGAACACCACA. T-BET: sense, CAACAACCCCTTTGCCAAAG; antisense, TCCCCCAAGCAGTTGACAGT; probe, CCGGGAGAACTTTGAGTCCATGTACGC. TNF- $\alpha$ : sense, CATCTTCTCAAAATTCGAGTGACAA; antisense, TGGGAGTAGACAAGGTACAACCC; probe, CACGTCGTAGCAAACCACCAAGTGGA.

PCR was performed on an iCycler with optical unit (Bio-Rad Laboratories Inc.). The PCR conditions were 5 minutes of denaturing at $95^{\circ} \mathrm{C}$ followed by 40 cycles of 15 seconds at $95^{\circ} \mathrm{C}$ and 45 seconds at $63^{\circ} \mathrm{C}$. Amplicon accumulation was measured during the annealing phase. Reaction efficiency for each primer was $100 \% \pm 10 \%$, based on the slope generated by standard curves using specific cloned amplicons. A standard curve was generated for each 96well plate using a plasmid containing the $\beta$-actin amplicon. Data were analyzed using iCycler analysis software version 2.3.

\section{Results}

p40-deficient mice display decreased EAE severity upon adoptive transfer of $M O G$-reactive cells. We and others have previously shown that $p 40^{-/-}$and $p 19^{-/-}$, but not $p 35^{-/-}$mice, are completely resistant to the induction of EAE upon active immunization with myelin antigen (1-3). Therefore, whether one examines inflam-

\section{Figure 4}

mation in $p 40^{-/-}$or $p 19^{-/-}$mice, the phenotype is exclusively due to the lack of IL-23. We wanted to extend these studies to assess the role and function of p40 during the effector phase of EAE. In order to do so, we adoptively transferred MOG-reactive lymphocytes (generated in WT mice) into either $p 40^{-/-}$or WT mice. Figure 1 and Table 1 show that $p 40^{-/-}$mice develop EAE with significantly delayed kinetics and decreased severity $(P<0.01)$, indicating that hostexpressed p40 is involved in the maintenance of encephalitogenicity during the effector phase of EAE. These data are consistent with the observations of Cua and colleagues have shown that intracerebral administration of IL-23 after disease onset can restore disease in $p 19^{-/-}$mice (3).

p40 drives encephalitogenicity but not inflammation. In spite of the reduced clinical phenotype of $p 40^{-/-}$mice upon adoptive transfer of MOG-reactive lymphocytes, histological analysis (Figure 2 ) revealed significant inflammation in the spinal cords of $p 40^{-/-}$mice that was comparable to that of WT mice with significantly more severe EAE. This finding supports the notion that $\mathrm{p} 40$ is not absolutely required for the infiltration of inflammatory cells into the CNS, but rather alters the degree of encephalitogenicity upon entry into the CNS. For a more quantitative analysis

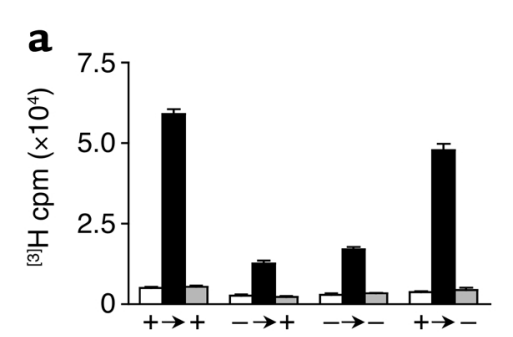

C

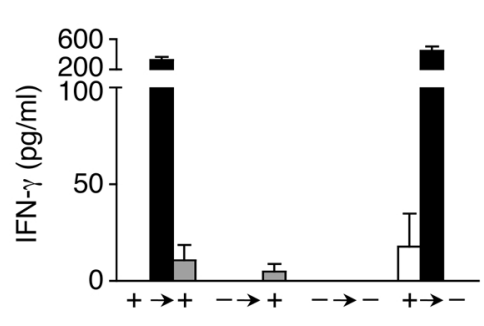

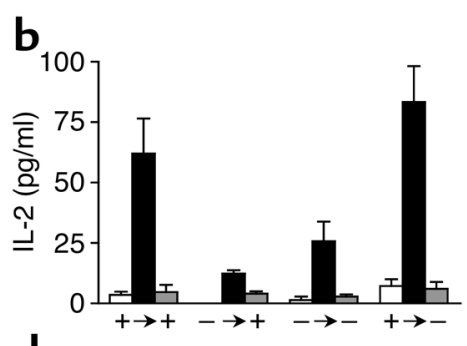

d

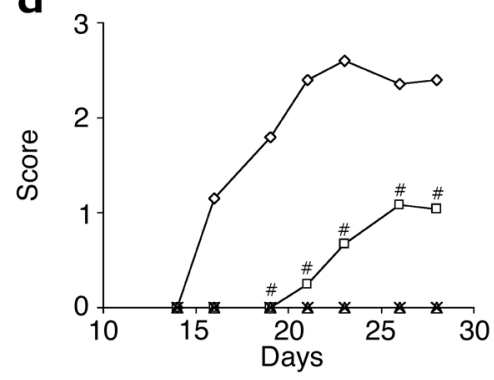

BM transfer fully reconstitutes the host's ability to drive Th1 immunity. After 2 months of recovery, BM chimeric mice were immunized with MOG peptide in CFA and lymph nodes were removed 5 days later. Recall proliferation (a), IL-2 (b), and IFN- $\gamma$ secretion (c) in response to either $100 \mu \mathrm{g}$ MOG peptide or irrelevant myelin proteolipid protein peptide was assessed in vitro. White bars, medium alone; black bars, $100 \mu \mathrm{g}$ MOG peptide; gray bars, $100 \mu \mathrm{g}$ PLP peptide. +, WT; -, $p 40^{-/-}$; arrows indicates the transfer of BM. (d) After BM transplant, mice (diamonds, $p 40^{+/+} \rightarrow$ $p 40^{+/+}$; squares, $p 40^{+/+} \rightarrow p 40^{-/-} ; \times, p 40^{-/-} \rightarrow p 40^{+/+}$; triangles, $p 40^{-/-} \rightarrow p 40^{-/^{-}}$) were allowed to recover for 2 months, then immunized with $\mathrm{MOG}_{35-55}$ in $\mathrm{CFA}$ as described and scored for clinical disease. In all experiments, the disease score between the susceptible $p 40^{+/+} \rightarrow p 40^{+/+}$and $p 40^{+/+} \rightarrow p 40^{-/-}$mice was significantly different as assessed using a nonpaired Student's $t$ test $\left({ }^{\#} P<0.01\right)$. Shown is a representative of four individual experiments. 
Table 3

EAE in BM chimeras induced by active immunization with MOG peptide (see Figure 4d)

\begin{tabular}{lcccc}
\hline Group & \multicolumn{2}{c}{ Incidence } & Mean day of disease onset $^{\mathrm{A}}$ & Mean maximal clinical score $^{\mathrm{A}}$ \\
$\mathrm{WT} \rightarrow \mathrm{WT}$ & $4 / 5$ & $80 \%$ & 17.2 & 4 \\
$\mathrm{WT} \rightarrow \mathrm{IL}-12 p 40^{-/-}$ & $4 / 6$ & $67 \%$ & 23.3 & 2.1 \\
$\mathrm{IL}-12 p 40^{-/-} \rightarrow \mathrm{IL}-12 p 40^{-/-}$ & $0 / 3$ & $0 \%$ & \\
$\mathrm{IL}-12 p 40^{-/-} \rightarrow \mathrm{WT}$ & $0 / 4$ & $0 \%$ & & \\
& &
\end{tabular}

${ }^{A}$ Diseased animals only.

of CNS infiltrates, 5 days after disease onset, the recipient mice were euthanized, and infiltration into the spinal cord was measured by flow cytometry. Figure 3 a shows that the absence of $\mathrm{p} 40$ in the recipient does not significantly alter the degree of inflammation. Both WT and $p 40^{-/-}$mice immunized with MOG $_{35-55}$ peptide display a high number of $\mathrm{CD} 45^{\mathrm{hi}}$ infiltrating cells compared with nonimmunized mice. We did not observe an overt change in the cellular profile of infiltrating cells with regard to the percentage of $\mathrm{CD}^{+}$or $\mathrm{CD}^{+} \mathrm{T}$ cells, macrophages, or polymorphonuclear cells as assessed by flow cytometry (Figure $3 \mathrm{~b}$ ). The number of NK cells or NK T cells was negligible as assessed by NK1.1 staining (not shown). The data indicate that the quality of the CNS immune response is different in the WT than in the $p 40^{---} \mathrm{CNS}$, but that the differences do not lie in the composition of the infiltrating cells.

Production of $p 40$ CNS-endogenous cells is critical for the induction of EAE upon active immunization. We have previously shown that CNS-endogenous cells, most likely microglia, control the extent of inflammation in EAE via CD40 (11). Mice in which CD40 is not expressed within the CNS show a decrease in inflammatory infiltrates and a paucity in early chemokine expression by CNS-resident cells. In order to assess the role of $\mathrm{p} 40$ expressed by cells within the CNS during the effector phase of EAE, we selectively expressed p40 within the CNS using irradiation BM chimeras. Following irradiation and $\mathrm{BM}$ reconstitution, the peripheral APC compartment is comprised entirely of BM-donor-derived cells, whereas $100 \%$ of CNS-resident microglia remain of host origin (11). We generated $\mathrm{BM}$ chimeras by transferring $p 40^{-/-}$or WT BM into irradiated $p 40^{-/-}$and WT recipients (Table 2). To confirm the phenotype of the reconstituted peripheral APC compartment, we immunized these mice and determined the capacity of APCs in the periphery to support Th1 responses in a recall assay. Figure 4, a and $b$, shows that the antigen-induced $T$ cell proliferation and production of IL- 2 and IFN- $\gamma$ are intact in $\mathrm{WT} \rightarrow \mathrm{WT}$ and WT $\rightarrow p 40^{-/-}$mice. In contrast, in $p 40^{-/-} \rightarrow \mathrm{WT}$ and $p 40^{-/-} \rightarrow p 40^{-/-}$mice, $\mathrm{T}$ cells were incapable of inducing IFN- $\gamma$ secretion.

Using this panel of mixed $\mathrm{BM}$ chimeric mice, mice were immunized with $\mathrm{MOG}_{35-55}$ in CFA and disease development was assessed as described. Figure $4 \mathrm{~d}$ and Table 3 show that 440 produced by cells resident to the
CNS is essential for full disease development. In the absence of CNS-derived p40 (WT $\rightarrow p 40^{-/-}$), we saw a marked delay in disease onset and a decrease in disease severity. It is important to emphasize that while both $\mathrm{WT} \rightarrow p 40^{+/+}$and WT $\rightarrow p 40^{-/-}$chimeras have an equivalent capacity to generate peripheral Th1-type $\mathrm{T}$ cells, the disease in the latter is compromised.

Th1 polarization by CNS-derived IL-12 $p 40$. We wanted to determine the impact of $\mathrm{p} 40$ expressed within the CNS on the cytokine profile of the infiltrating lymphocytes. We immunized $p 40^{-/-}$BM chimeras with $M_{35-55}$ in CFA as described. The mice were sacrificed at the first sign of clinical disease to extract RNA from the spinal cords. Expression levels of Th1 and Th2 markers were assessed by real-time PCR using hydrolysis probes (TaqMan) (Figure 5). The data reveal that the infiltrates in $p 40^{-/-}$mice display a Th2 polarized phenotype, whereas the WT mice appear more Th1 biased. A similar trend could be observed in $p 40^{-/-}$mice upon adoptive transfer (data not shown). The data indicate that the encephalitogenic potential of infiltrating lymphocytes is nurtured by $\mathrm{p} 40$ expressed within the CNS.

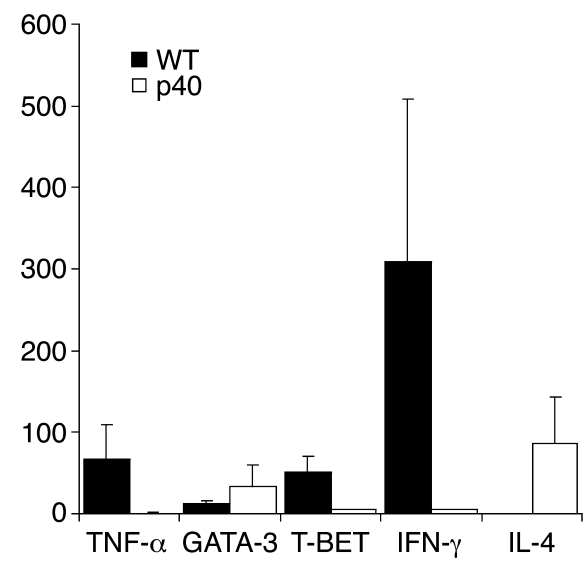

Figure 5

Expression profile of inflamed spinal cords. RNA was isolated as described from inflamed spinal cords 24 days after immunization. Real-time RT-PCR analysis was performed by measuring amplicon accumulation using TaqMan probes (Qiagen Inc.) (black bars, WT; white bars, $\left.p 40^{--}\right)$. Data are expressed as arbitrary units based on the standard curve of triplicate wells \pm SD. Shown is a representative of at least three individual experiments. Levels of $\beta$-actin mRNA were similar in both samples, and other mRNA species levels were normalized to $\beta$-actin. 


\section{Discussion}

The goal of our study was to determine the role of CNS-expressed p40 during the effector phase of EAE. Upon adoptive transfer of fully primed and activated WT MOG-reactive lymphocytes into $p 40^{-/-}$mice, we observed a marked delay in disease onset and a significant decrease of disease severity when compared with WT mice. Interestingly, although the degree of clinical symptoms was modest, the spinal cords of these mice still showed a large number of inflammatory infiltrates. Upon entry of leukocytes into the CNS, resident cells, in particular microglial cells, become activated and secrete a variety of proinflammatory factors including IL-12 and IL-23 $(3,9)$. We hypothesized that it is the CNS microenvironment that regulates $\mathrm{T}$ cell behavior during CNS inflammation in both EAE and MS. We generated BM chimeras in order to assess the role of p40 expressed by CNS-endogenous cells (radioresistant compartment), while having an immune compartment fully capable of supporting $\mathrm{T}$ cell activation, Th1 polarization, and encephalitogenicity. After active immunization of these mice, in the absence of $\mathrm{p} 40$ expressed within the CNS, we observed only subclinical EAE. In the absence of p 40 from the CNS, autoreactive lymphocytes still infiltrate the CNS, but do not fully develop their encephalitogenic potential. This finding is consistent with a recent report showing that mice deficient in the p19 subunit of IL-23 develop clinical EAE only upon delivery of IL-23 into the CNS. Peripheral complementation was insufficient to break EAE resistance (3). Furthermore, CNSinfiltrating inflammatory macrophages have been shown to express p19 and thus IL-23 at levels comparable to those expressed by CNS-resident microglia (3). However, the amount of IL-23 produced by CNSinvading cells alone is insufficient to drive a fulminant encephalitogenic Th1 response. It appears that the CNS residents participate in Th polarization and that this participation is a critical determinant in the disease pathogenesis.

To assess the impact of $\mathrm{p} 40$ secretion by CNS-resident cells on the inflammatory response, we evaluated the expression profile of Th1/2-associated genes expressed by CNS-infiltrating $\mathrm{T}$ cells. Expression analysis of spinal cords for Th1 and Th2 markers revealed that in the absence of $\mathrm{p} 40$ from the CNS, a shift from a Th 1 profile toward a Th $2 / 0$ polarized phenotype was induced.

The Th1/2 paradigm of inflammation in EAE and MS is challenged by gene-deletion studies showing that EAE can develop irrespective of Th1 cytokines (12-16) and Th1-inducing cytokines (e.g., IL-12). Thus, one cannot assume that the expression of Th 1 markers correlates with encephalitogenicity. Nonetheless, it appears that continued exposure of lymphocytes to IL-23 at the site of inflammation is critical for the sustained expression of Th1-associated genes. As for MS, therapeutic intervention by targeting the IL-12/23 pathway may provide a powerful inhibitor of disease progression without the side effects of broad immune suppression.

\section{Acknowledgments}

This work was supported by a research grant from the National Multiple Sclerosis Society (RG-3323A1/T to B. Becher) and an NIH grant (AI-49580-01 to R.J. Noelle). B. Becher is a Harry Weaver Neuroscience Scholar of the National Multiple Sclerosis Society.

1. Becher, B., Durell, B.G., and Noelle, R.J. 2002. Experimental autoimmune encephalitis and inflammation in the absence of interleukin-12. J. Clin. Invest. 110:493-497. doi:10.1172/JCI200215751.

2. Gran, B., et al. 2002. IL-12p35-deficient mice are susceptible to experimental autoimmune encephalomyelitis: evidence for redundancy in the IL-12 system in the induction of central nervous system autoimmune demyelination. J. Immunol. 169:7104-7110.

3. Cua, D.J., et al. 2003. Interleukin-23 rather than interleukin-12 is the critical cytokine for autoimmune inflammation of the brain. Nature. 421:744-748.

4. Oppmann, B., et al. 2000. Novel p19 protein engages IL-12p40 to form a cytokine, IL-23, with biological activities similar as well as distinct from IL-12. Immunity. 13:715-725.

5. Gately, M.K., et al. 1998. The interleukin-12/interleukin-12-receptor system: role in normal and pathologic immune responses. Annu. Rev. Immunol. 16:495-521.

6. Windhagen, A., et al. 1995. Expression of costimulatory molecules B7-1 (CD80), B7-2 (CD86), and interleukin 12 cytokine in multiple sclerosis lesions. J. Exp. Med. 182:1985-1996.

7. Issazadeh, S., Navikas, V., Schaub, M., Sayegh, M., and Khoury, S. 1998. Kinetics of expression of costimulatory molecules and their ligands in murine relapsing experimental autoimmune encephalomyelitis in vivo. J. Immunol. 161:1104-1112.

8. Aloisi, F., Penna, G., Polazzi, E., Minghetti, L., and Adorini, L. 1999. CD40-CD154 interaction and IFN-gamma are required for IL-12 but not prostaglandin E2 secretion by microglia during antigen presentation to Th1 cells. J. Immunol. 162:1384-1391.

9. Becher, B., Prat, A., and Antel, J.P. 2000. Brain-immune connection: immuno-regulatory properties of CNS-resident cells. Glia. 29:293-304.

10. Becher, B., Dodelet, V., Fedorowicz, V., and Antel, J.P. 1996. Soluble tumor necrosis factor receptor inhibits interleukin 12 production by stimulated human adult microglial cells in vitro. J. Clin. Invest. 98:1539-1543

11. Becher, B., Durell, B.G., Miga, A.V., Hickey, W.F., and Noelle, R.J. 2001. The clinical course of experimental autoimmune encephalomyelitis and inflammation is controlled by the expression of CD40 within the central nervous system. J. Exp. Med. 193:967-974.

12. Korner, H., et al. 1997. Critical points of tumor necrosis factor action in CNS autoimmune inflammation defined by gene targeting. J. Exp. Med. 186:1585-1590.

13. Chu, C.Q., Wittmer, S., and Dalton, D.K. 2000. Failure to suppress the expansion of the activated CD4 $\mathrm{T}$ cell population in interferon gammadeficient mice leads to exacerbation of experimental autoimmune encephalomyelitis. J. Exp. Med. 192:123-128.

14. Willenborg, D.O., Fordham, S., Bernard, C.C., Cowden, W.B., and Ramshaw, I.A. 1996. IFN-gamma plays a critical down-regulatory role in the induction and effector phase of myelin oligodendrocyte glycoproteininduced autoimmune encephalomyelitis. J. Immunol. 157:3223-3227.

15. Krakowski, M., and Owens, T. 1996. Interferon-gamma confers resistance to experimental allergic encephalomyelitis. Eur. J. Immunol. 26:1641-1646.

16. Frei, K., et al. 1997. Tumor necrosis factor alpha and lymphotoxin alpha are not required for induction of acute experimental autoimmune encephalomyelitis. J. Exp. Med. 185:2177-2182. 